\title{
Observational study of generation conditions of substorm-associated low-frequency AKR emissions
}

\author{
A. Olsson ${ }^{1}$, P. Janhunen ${ }^{2}$, J. Hanasz ${ }^{3}$, M. Mogilevsky ${ }^{4}$, S. Perraut ${ }^{5}$, and J. D. Menietti ${ }^{6}$ \\ ${ }^{1}$ Swedish Institute of Space Physics, 75591 Upsala, Sweden \\ ${ }^{2}$ Finnish Meteorological Institute, Space Research, POB 503, FIN-00101, Helsinki, Finland \\ ${ }^{3}$ Space Research Centre, ul. Rabianska 8, 87-100 Torun, Poland \\ ${ }^{4}$ Space Research Institue, Russian Academy of Sciences, Profsoyuznaya 84/32, Moscow, 117 810, Russia \\ ${ }^{5}$ Centre d'Etude des Environments Terrestre et Planetaires/Centre National de la Recherche Scientifique/Universite de \\ Versailles-Saint Quentin, Velizy, France \\ ${ }^{6}$ University of Iowa, Iowa City, Iowa 52242-1479, USA
}

Received: 28 October 2003 - Revised: 5 March 2004 - Accepted: 21 April 2004 - Published: 3 November 2004

\begin{abstract}
It has lately been shown that low-frequency bursts of auroral kilometric radiation (AKR) are nearly exclusively associated with substorm expansion phases. Here we study low-frequency AKR using Polar PWI and Interball POLRAD instruments to constrain its possible generation mechanisms. We find that there are more low-frequency AKR emission events during wintertime and equinoxes than during summertime. The dot-AKR emission radial distance range coincides well with the region where the deepest density cavities are seen statistically during $K_{p}>2$. We suggest that the dot-AKR emissions originate in the deepest density cavities during substorm onsets. The mechanism for generating dot-AKR is possibly strong Alfvén waves entering the cavity from the magnetosphere and changing their character to more inertial, which causes the Alfvén wave associated parallel electric field to increase. This field may locally accelerate electrons inside the cavity enough to produce low-frequency AKR emission. We use Interball IESP low-frequency wave data to verify that in about half of the cases the dot-AKR is accompanied by low-frequency wave activity containing a magnetic component, i.e. probably inertial Alfvén waves. Because of the observational geometry, this result is consistent with the idea that inertial Alfvén waves might always be present in the source region when dot-AKR is generated. The paper illustrates once more the importance of radio emissions as a powerful remote diagnostic tool of auroral processes, which is not only relevant for the Earth's magnetosphere but may be relevant in the future in studying extrasolar planets.
\end{abstract}

Key words. Magnetospheric physics (storms and substorms; auroral phenomena) - Space plasma physics (waveparticle interaction)

Correspondence to: A. Olsson

(ao@irfu.se)

\section{Introduction}

Auroral kilometric radiation (AKR) is an intense radio emission from the auroral acceleration region (Benediktov et al., 1965; Gurnett, 1974; Kurth et al., 1975) usually between 50 and $400 \mathrm{kHz}$ (Gurnett, 1991). While the quoted frequency limit is rather typical, sometimes the frequency goes up to $700-800 \mathrm{kHz}$. The generation mechanism for AKR is thought to be the cyclotron maser instability, whose free energy source is a perpendicular positive slope in the velocity distribution of hot (some $\mathrm{keV}$ ) electrons (Wu and Lee, 1979). The positive slope could be associated with either the loss cone or the electron shell (horseshoe) distribution. The latter mechanism has been suggested to be more efficient (Strangeway et al., 2001). The electron shell distribution is presumably created by an upward parallel electric field existing at the bottom of a negative potential structure associated with a discrete auroral arc. Only a fraction of the electron shell distribution energy goes into producing AKR, the rest of the energy is dissipated in the ionosphere by the precipitating inverted- $\mathrm{V}$ electrons. Just above the bottom of the potential structure the plasma density is low, thus the mechanism is consistent with the observation that AKR is mainly generated in regions of low plasma density (Benson and Calvert, 1979; Calvert, 1981a; Hilgers, 1992). In this paper we make the working hypothesis that the cyclotron maser instability is the correct theory for AKR emissions and that whenever electrons are accelerated strongly enough, in the parallel direction, AKR emissions follow either directly or after the mirror force has converted an initially beam-like distribution into a horseshoe or shell distribution containing perpendicular positive slopes (Benson and Calvert, 1979).

The frequency of AKR is nearly equal to the local electron gyrofrequency of the generation region $(f=28 B$ when $f$ is in $\mathrm{Hz}$ and $B$ is in $\mathrm{nT}$ ). Being electromagnetic radiation, AKR 
propagates almost straight in the emission cone and thus can be used for remote sensing. Since the altitude dependence of the magnetic field is well known, the AKR generation altitude can be estimated from the AKR frequency. The usual AKR frequencies $(100-400 \mathrm{kHz})$ correspond to altitudes of $3300-9000 \mathrm{~km}\left(1.5-2.5 R_{E}\right.$ radial distance), which is in accordance with the idea that AKR is produced within the acceleration region (Roux et al., 1993; Strangeway et al., 2001). AKR propagates upward from the generation region in a cone centred along the magnetic field direction. The half-angle of the cone varies between 20 and $65 \mathrm{deg}$, depending on the frequency (Calvert (1981b), Fig. 3).

Wideband bursts of AKR (WBB-AKR) are associated with substorms' expansion phases and "manifested by a sudden commencement $(<1 \mathrm{~min})$ of the emission over a wide frequency range, followed by a decay lasting $\sim 10 \mathrm{~min}$ or more" (Hanasz et al., 2001). Their source regions may reside in the western part of the auroral bulges occurring in the premidnight sector, i.e. the region containing the brightest aurora during substorm expansion phase (de Feraudy et al., 2001). In many of the WBB-AKR events the frequencies extend well below $100 \mathrm{kHz}$, corresponding to an emission altitude above $9000 \mathrm{~km}$. In many AKR spectra there is a shortlived island of emissions around $50 \mathrm{kHz}$ which is disconnected from the main emissions at higher frequencies. Following de Feraudy et al. (2001), we call it the dot-AKR. The term "exclamation point AKR" employed by Hanasz et al. (2001) refers to the same phenomenon.

In this paper we use the following terminology. Any AKR emission occurring below $100 \mathrm{kHz}$ will be called lowfrequency AKR (LFAKR). The term dot-AKR is used for the subclass of LFAKR where there is a disconnected island of emission in the time-frequency spectrogram at $\sim 50 \mathrm{kHz}$. Finally, LFAKR which is not dot-AKR will be called smooth low-frequency AKR (SLFAKR), i.e. any emission below $100 \mathrm{kHz}$ that varies smoothly and continuously with frequency is SLFAKR. Notice that these definitions refer only to the phenomenological features of the emissions.

In the same frequency range (from the local gyro frequency to $100 \mathrm{kHz}$ ) where the LFAKR occurs, several other freely propagating radiation components have been reported and named in the literature. The nonthermal continuum radiation (Gurnett, 1975) is mainly a dayside phenomenon generated between 5 and $8 R_{E}$ and is thus probably not related to the LFAKR discussed in this paper. The low-frequency radiation (LFR) discussed by Filbert and Kellogg (1989), and especially the nonthermal continuum enhancement radiation or the auroral myriametric radiation (AMR) of Hashimoto et al. (1998) are possibly at least partly the same as our LFAKR. The isotropic terrestrial kilometric radiation (ITKR) observations made in the Lagrange point by Steinberg et al. (1990) may have at least partly the same source as our LFAKR, but the directional properties of the radiation have been heavily modified by propagation effects in the dense solar wind. In short, a nightside emission occurring between the local gyrofrequency and about $100 \mathrm{kHz}$ is LFAKR if it is sporadic in nature and occurs together with intense AKR but not neces- sarily having a detailed temporal correlation with it. Depending on where the observations are made, propagation effects depending on the plasma density structure may modify the picture.

The goal of this paper is to study the basic properties of LFAKR and their relation to the auroral cavities. The association of normal AKR with cavities is already well known (Louarn and LeQueau, 1996a, b). We determine altitudes of the LFAKR sources and their seasonal variations, and association of the LFAKR with the occurrence of the lowfrequency wave activity. We will also present a scenario for the generation conditions of LFAKR. In Sect. 3 we statistically find that the sources of the LFAKR occur at times of increased substorm activity, within cavities formed at altitudes about $3 R_{E}$ at auroral latitudes and evening local times. In Sect. 4 we show evidence that the occurrence of LFAKR is associated with the Alfvénic wave activity. A similar analysis was briefly described by Hanasz et al. (2001), however, without having the explicit requirement that the Interball footpoint is within the UVI auroral bulge. Alfvén waves at lower altitude (in the acceleration region and below) have been analysed, for example, by Chaston et al. (2002) and Volwerk et al. (1996). Above $4 R_{E}$ geocentric distance, they have been shown to be important in auroral energetics (Keiling et al., 2002, 2003).

\section{Instrumentation}

\subsection{Polar PWI}

The plasma wave instrument (PWI) on board the Polar satellite (Gurnett et al., 1995) measures electric and magnetic waves in the frequency range $26 \mathrm{~Hz}-800 \mathrm{kHz}$. The instrument worked from 27 February 1996, until 16 September 1997. During that period, Polar spent most of its time at a distance of 4-9 $R_{E}$ above the Northern Hemisphere and thus could observe AKR in this region. Polar PWI has previously been used for studying AKR, for example, by Imhof et al. (1998). In this study we use the SFR-A subinstrument for measuring AKR.

\subsection{Polar UVI}

The ultraviolet imager (UVI) on board Polar images the auroral emissions in the far ultraviolet with a nominal time resolution of $37 \mathrm{~s}$ (Torr et al., 1995). We use UVI for checking if and where a substorm expansion is preceeding during the events.

\subsection{Interball auroral probe (POLRAD)}

The Interball Auroral Probe (Interball-2) was launched on 29 August, 1996, to an orbit with apogee $19140 \mathrm{~km}$ and perigee $780 \mathrm{~km}$, and an inclination to the equator of $62 \mathrm{de}-$ grees. The Interball Auroral Probe flew somewhat above the source region of LFAKR. POLRAD was a high-frequency 
wave instrument on board Auroral Probe, capable of measuring power density of the wave electric component in a frequency range from 4 to $1000 \mathrm{kHz}$ (Hanasz et al., 1998, 2001). POLRAD worked from 4 September 1996 to 26 January 1999.

\subsection{Interball auroral probe (IESP)}

IESP is a low-frequency wave instrument on board the Interball Auroral Probe which can measure all six components of the electromagnetic field in the $0.1-30 \mathrm{~Hz}$ frequency range (Perraut et al., 1998). It worked from 7 September 1996, until 28 October 1998, however, the magnetic channels stopped working on 20 December 1996. We use IESP for estimating the ratio of the electric and magnetic wave components to answer the question of whether the observed waves are Alfvénic in nature or not.

\subsection{How data sets are used}

In Sect. 3 we use only Polar data. Polar is at high altitude and can thus almost always observe AKR no matter where in the auroral region the emissions are located. Polar also has the UVI camera for checking the connection to optical emissions. The drawback of Polar is that when it observes AKR with PWI and auroral UV emission with UVI, the spacecraft almost never resides on auroral field lines. In other words, Polar almost never sees its own footprint. Therefore, in Sect. 4 we use Interball Auroral Probe data to complement Polar. Interball provides in situ wave and AKR data from within close vicinity of the AKR source field lines.

\section{Seasonal properties of LFAKR}

We have analysed all Polar PWI data to find LFAKR signatures. The data set starts in early March, 1996, and ends on 15 September 1997, thus it covers two summers and one winter season in the Northern Hemisphere. The search used the following automated criteria:

1. Polar must be in the nightside magnetosphere (18:0006:00 MLT) at larger than $5 R_{E}$ radial distance, to be sure that we are above the generation altitude of LFAKR.

2. For AKR the $E / B$ ratio is equal to $c$, thus we required that $E / B \leq 5 \times 10^{8} \mathrm{~m} / \mathrm{s}$ as measured by PWI SFR-A.

3. The electric spectral power somewhere in the 30 $50 \mathrm{kHz}$ frequency range must exceed $10^{-14}(\mathrm{~V} / \mathrm{m})^{2} / \mathrm{Hz}$.

The resulting events were then manually checked for data errors. We also checked that the local gyro and plasma frequencies were lower than the LFAKR frequency. The search yielded 149 LFAKR events, 62 of which occurred with UVI data which was also available. Of these 62 events, 59 were associated with a clear auroral breakup as determined from UVI. The good correlation of LFAKR with auroral breakups

\section{LFAKR events}

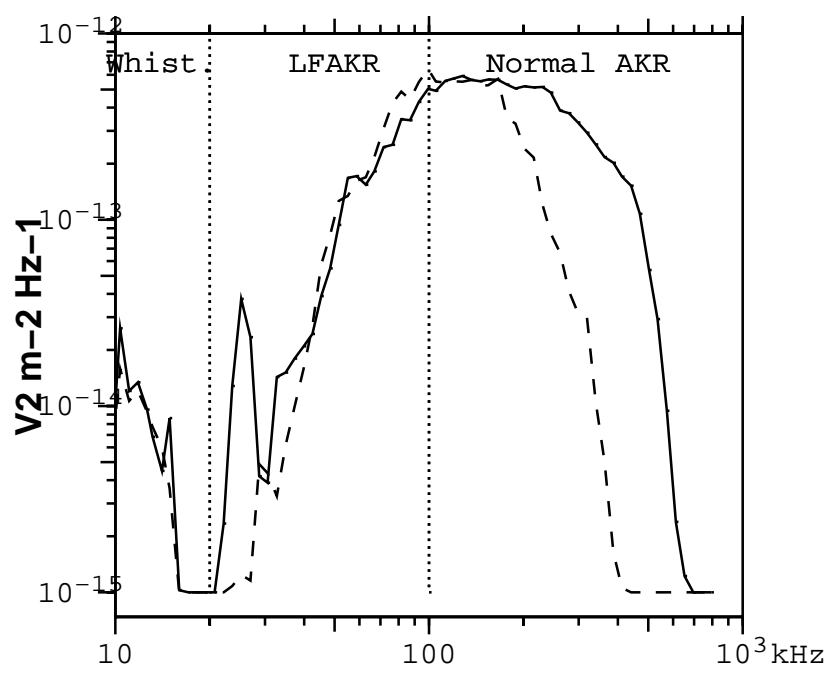

Fig. 1. Arithmetically averaged power density of AKR for lowfrequency AKR events (LFAKR) plotted as a function of frequency, obtained from Polar PWI measurements. Solid line corresponds to winter months (November-February) and dashed line to summer months (May-August). Most of the plot corresponds to normal AKR, while LFAKR contribution is seen in the frequency range $30-50 \mathrm{kHz}$. Power in the range $10-15 \mathrm{kHz}$ is not AKR but due to whistler wave activity since its frequency is below the local electron gyrofrequency. The peak at $25 \mathrm{kHz}$ is due to a single strong dot-AKR event at this frequency (18 November 1996, 14:37 UT).

is consistent with previous studies (de Feraudy et al., 2001; Hanasz et al., 2001).

For the 149 LFAKR events mentioned, we determined the boundaries of significant $30-50 \mathrm{kHz}$ emission manually. Over these times, an arithmetic average of the PWI SFR-A electric power density was carried out. Winter month events (November-February) and summer events (May-August) were averaged separately and events which occurred in March, April, September or October (equinox events) were ignored. The number of winter events was 47 , the number of summer events 23 , and the number of ignored equinox events 79. Since the data set covers three equinoxes, one winter and two summers, the numbers indicate that LFAKR events are about equally common in winter and equinox $(\sim 12$ events per month), but more rare during summertime $(\sim 3$ events per month). Notice that there is an equal number of substorms in summertime and wintertime (since substorms are global and when one hemisphere is in winter the other is in summer), so the reason for the rarity of LFAKR events during summertime must reflect some change in the local plasma environment rather than a lack of a suitable magnetospheric driver during summertime. We shall give a possible explanation for this anomaly in the Discussion section. The resulting time-averaged power density during LFAKR is shown in Fig. 1, where the solid line is for winter months and the dashed line for summer months. 


\section{LFAKR events}

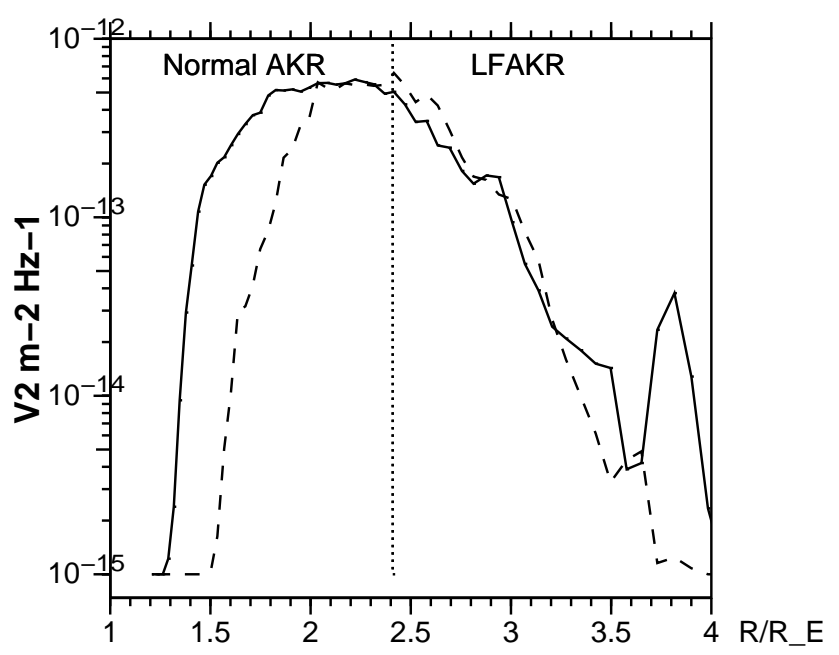

Fig. 2. Same as Fig. 1 but plotted as a function of AKR source radial distance.

In Fig. 2 we show the same data but now the horizontal axis is the radial distance $R$ of the generation region instead of the measured frequency. The frequency has been turned to radial distance by assuming that the magnetic field at $R$ equals $B=50000 \mathrm{nT} /\left(R / R_{E}\right)^{3}$; this approximation is adequate for our purposes here.

Slightly outside the main topic of this paper but still interesting to note, we observe the following from Fig. 2 concerning high frequency AKR. The lowest AKR emissions occur at $1.5 R_{E}$ radial distance $(3000 \mathrm{~km}$ altitude) during summertime and about $1500 \mathrm{~km}$ lower during wintertime, i.e. that there is a seasonal shift. Notice that the events under consideration here are not just any AKR but they correspond to substorm expansion phases, during which the bottom of the acceleration region resides at lower altitude than when the AKR comes from quiet auroral arcs. This is seen, for example, when comparing low $K_{p}\left(K_{p} \leq 2\right.$, mostly non-substorm intervals) and high $K_{p}\left(K_{p}>2\right.$, substorm-rich intervals) occurrence frequencies of electric potential minima (Janhunen et al., 2004a) and auroral density depletions (Janhunen et al., 2002a). Indeed, it has been known for a long time that the acceleration region altitude depends on the season (Bennett et al., 1983). More recently, Kumamoto et al. (2003) showed that AKR frequency depends on season and also on solar cycle, in such a way that the altitude of intense AKR emissions is the lowest during wintertime solar minimum conditions.

Next, we manually classify the PWI events in dot-AKR and non-dot events which we call smooth low-frequency AKR (SLFAKR) events. Dot-AKR events are those where the low-frequency part causes an island which is more or less well separated from the main AKR and SLFAKR events. By SLFAKR we mean low-frequency emissions which do not form a discernable dot or island. Figure 3 shows an example of a dot-AKR event and Fig. 4 shows an example of a
19961214 Dot AKR

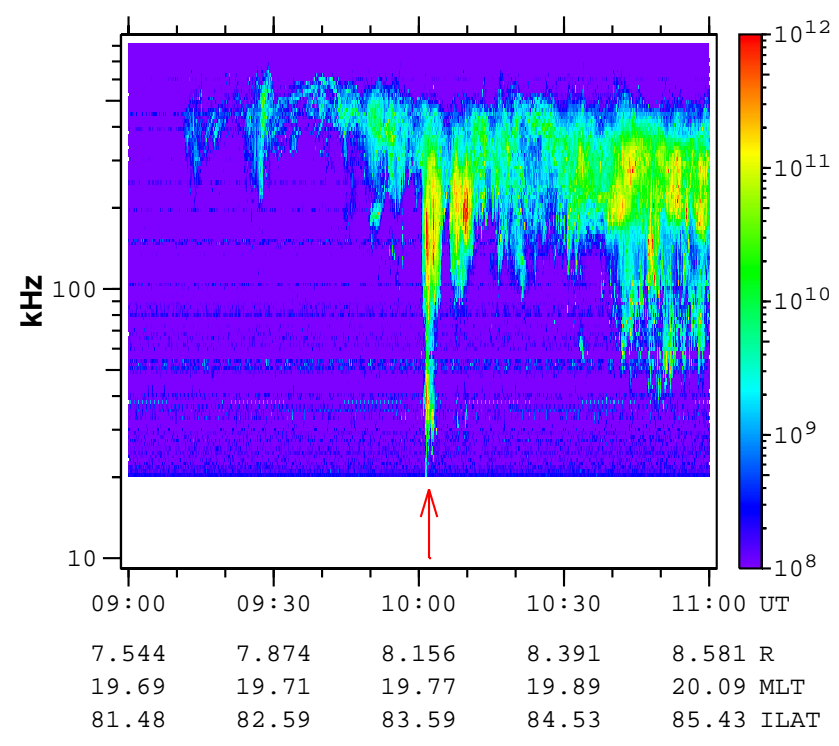

Fig. 3. Example of the radiospectrogram (in units of Jansky) of a dot-AKR event obtained on 14 December 1996 with the Plasma Wave Instrument (PWI) on Polar. The dot-AKR (red arrow) is seen near 10:00 UT at frequencies of $25-55 \mathrm{kHz}$.

\section{SLFAKR}

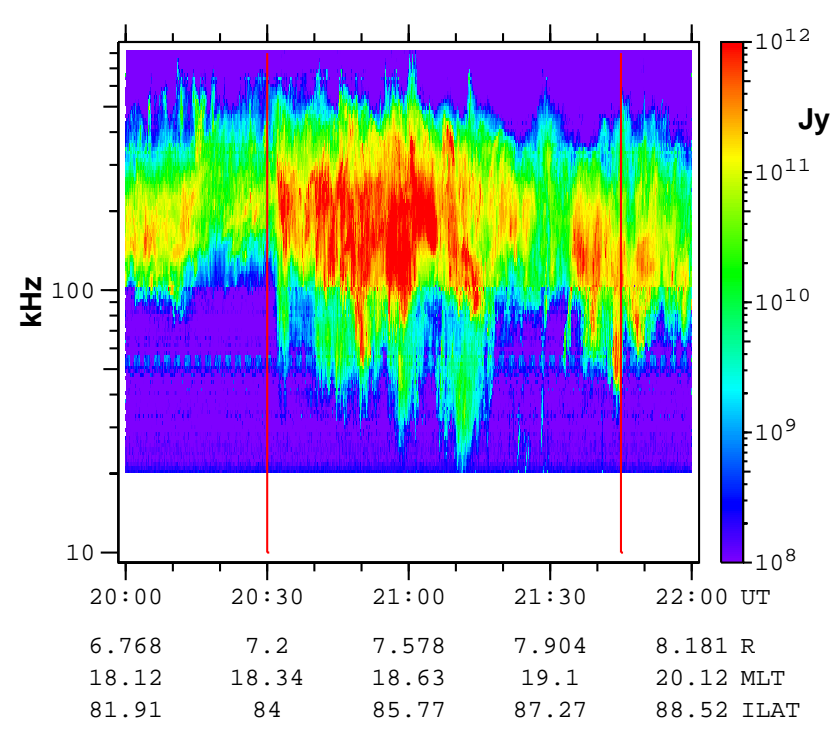

Fig. 4. Example of the radiospectrogram (in units of Jansky) of SLFAKR event obtained on 28 January 1997 with the Plasma Wave Instrument (PWI) on Polar. The event boundaries are marked with red vertical lines. In this paper we use the definition that all smooth AKR emissions below $100 \mathrm{kHz}$ are smooth low-frequency AKR (SLFAKR).

SLFAKR event. Figure 5 shows the histogram of the source region radial distance $R$ as determined from the frequency for dot-AKR and SLFAKR events. Summer and winter 

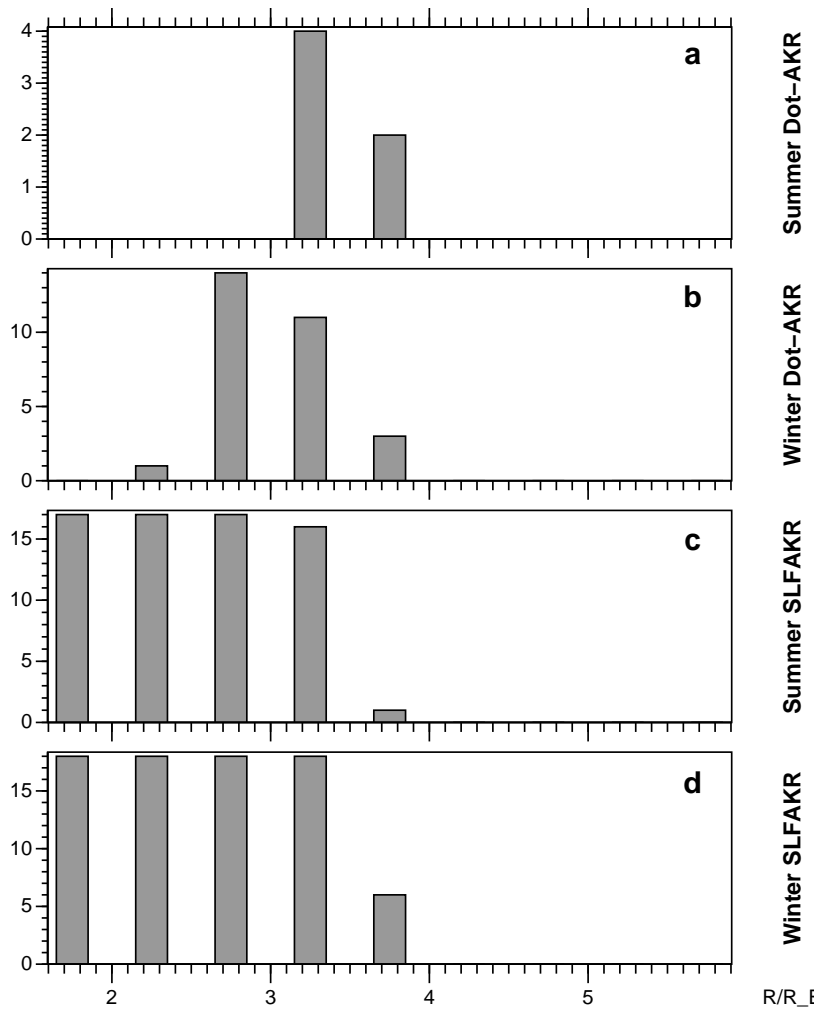

Fig. 5. Polar PWI data histogram of source region radial distance for (a) summer dot-AKR, (b) winter dot-AKR, (c) summer SLFAKR and (d) winter SLFAKR events. The SLFAKR panels show a cumulative sum up to the low cutoff frequency.

events are shown separately for both dot-AKR and SLFAKR. The quantity shown by the horizontal axis is the radial distance corresponding to the most intense emission in the dotAKR case and the low cutoff frequency in the SLFAKR case. The vertical axis is the number of events. For the SLFAKR events, a cumulative sum up to the low cutoff frequency is shown in the SLFAKR panels, i.e. basically all altitudes below the cutoff emit AKR in the SLFAKR case. We see that the dot-AKR events occur at higher altitude during summertime than during wintertime, while in the SLFAKR cutoff no seasonal effect can be discerned. The small statistics during summertime makes this conclusion not completely trustworthy. The application of Student's $t$-test shows that the probability that the mean altitudes during summer and winter are truly different is $85 \%$; in other words, the probability that the difference is accidental is $15 \%$.

The distinction of LFAKR events into dot-AKR and SLFAKR is necessarily somewhat subjective and also depends on the time resolution of the data (recall the phenomenological definitions from the fourth paragraph of the Introduction). Concerning possible physical mechanisms responsible for the phenomenological emission types dot-AKR and SLFAKR, our opinions are the following: 1) There is some specific transient physical mechanism which is responsible for dot-AKR. 2) The observed SLFAKR is a combi-
09-Dec-1996 14:43:10.779

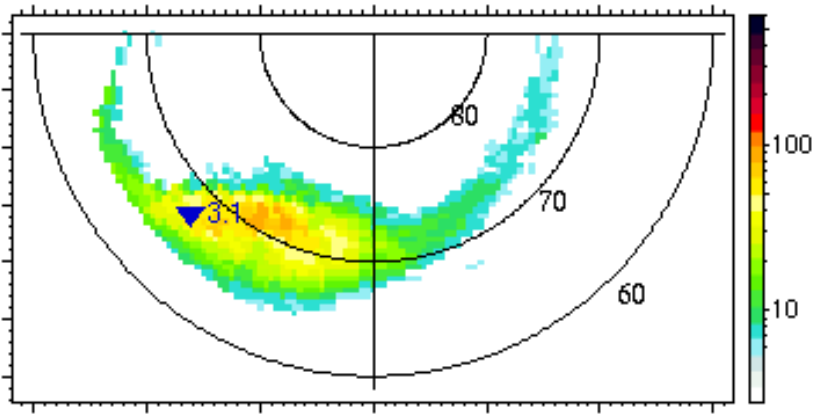

Fig. 6. Polar UVI data for 9 December 1996. The Interball Auroral Probe footpoint is marked with a triangle. Interball is making measurements in the auroral bulge. G. Parks and CDAWeb are acknowledged.

\section{MLT-ILAT}

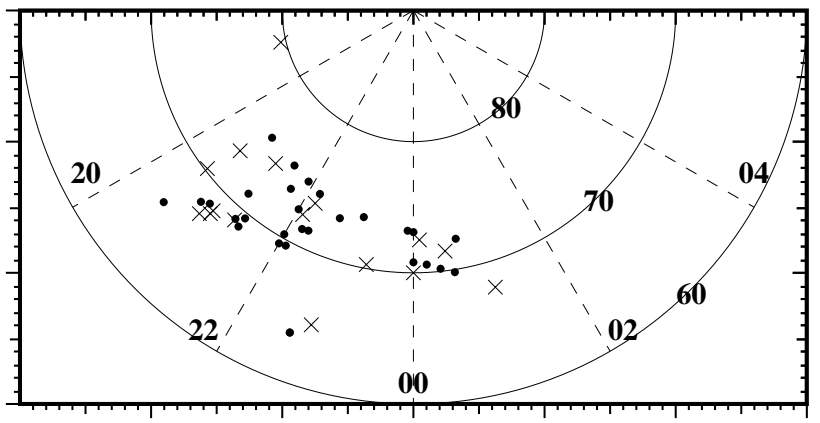

Fig. 7. Footprints of Interball Auroral Probe magnetic field lines at times of dot-AKR (dots) and SLFAKR (crosses) events.

nation of a) normal AKR that extends for some reason to lower frequencies than usually (for example, by increased ionospheric plasma density) and b) transient dot-AKR events superposed together so that their underlying "dot" character cannot be discerned from the data.

\section{IESP wave measurements near source region of dot- AKR and SLFAKR}

We selected dot-AKR and SLFAKR events where the Interball Auroral Probe footpoint is within an auroral bulge as judged from Polar UVI images. The events span the time interval 5 December 1996 until 9 December, 1997. An example is shown in Fig. 6. Again, the events were classified as dot-AKR and SLFAKR events, yielding 29 dot-AKR and 15 SLFAKR events. Figure 7 shows the MLT and ILAT of each Interball dot-AKR event (dots) and SLFAKR event (crosses). In accordance with earlier studies, the events mainly occur in the premidnight MLT sector which is typical for auroral bulges (Hanasz et al., 2001). 
The dot-AKR and SLFAKR events are summarised in Tables 1 and 2, respectively. For each event we list Interball radial distance, MLT, ILAT, the frequency range of the emission, the corresponding generation radial distance range, and letters telling whether low-frequency IESP electric (E) and magnetic (B) waves were detected simultaneously. A question mark indicates that the magnetic channel of IESP was not operational and a dash indicates that the corresponding signal was weak or nonexistent.

Among the dot-AKR events (Table 1), 6 out of 29 (21\%) cases have both electric and magnetic low-frequency IESP wave components significantly enhanced from the background level. In 7 cases (24\%) the electric wave component is enhanced but the magnetic part of IESP is not operating, so the existence of the magnetic wave component cannot be verified. Thus, in 13 cases $(45 \%)$ there is at least electric wave activity associated with the dot-AKR emission. For SLFAKR events (Table 2) the number with at least electric wave activity is 9 out of 15 , i.e. $60 \%$.

In Fig. 8 we show an example of POLRAD data (panel (d)) with IESP data (panels (a-c)). Panel (a) is the electric wave amplitude, panel (b) the magnetic wave amplitude, and panel (c) their ratio (the $E / B$ ratio). Notice that in the magnetic component there is rather high instrument noise level, so the $E / B$ ratio in panel (c) is meaningful only when clear discrete wave emissions are seen in panel (b). At times marked with an arrow there is an artificial calibration signal from another wave instrument (NVKONCH), so signals seen at these times do not correspond to physical waves.

\subsection{Wave mode seen in IESP data}

The frequency range considered $(0.2-10 \mathrm{~Hz})$ is well below the proton gyrofrequency, which is about $20 \mathrm{~Hz}$ in this case. Thus, the waves seen could in principle be either shear Alfvén waves, fast magnetosonic waves or ion acoustic waves. Further, if there is significant helium or oxygen content, oxygen or helium cyclotron and Bernstein modes could also exist in this frequency range. Ion acoustic and Bernstein waves have no significant magnetic component. Fast magnetosonic waves would have wavelengths of the order of an Earth radii, and since their phase velocity does not depend strongly on the magnetic field direction, they should propagate in all directions. However, this would contradict the observation that the waves are seen only on certain field lines. Thus, the waves are likely to be shear (inertial) Alfvén waves. Such waves have been extensively discussed in the literature for their possible role in accelerating auroral electrons (Hasegawa, 1976; Hui and Seyler, 1992; Lysak and Lotko, 1996). The perpendicular wave electric and magnetic field ratio, $E_{\perp} / B_{\perp}$, of inertial Alfvén waves is

$$
\frac{E_{\perp}}{B_{\perp}}=v_{A} \sqrt{1+k_{\perp}^{2} \lambda_{e}^{2}},
$$

where $v_{A}=B_{0} / \sqrt{\mu}_{0} \rho$ is the MHD Alfvén speed, $k_{\perp}$ the perpendicular wave number, $\lambda_{e}=c / \omega_{p e}$ the collisionless skin depth, $\omega_{p e}=\sqrt{n e^{2} /\left(\epsilon_{0} m_{e}\right)}$ the electron plasma angular fre-

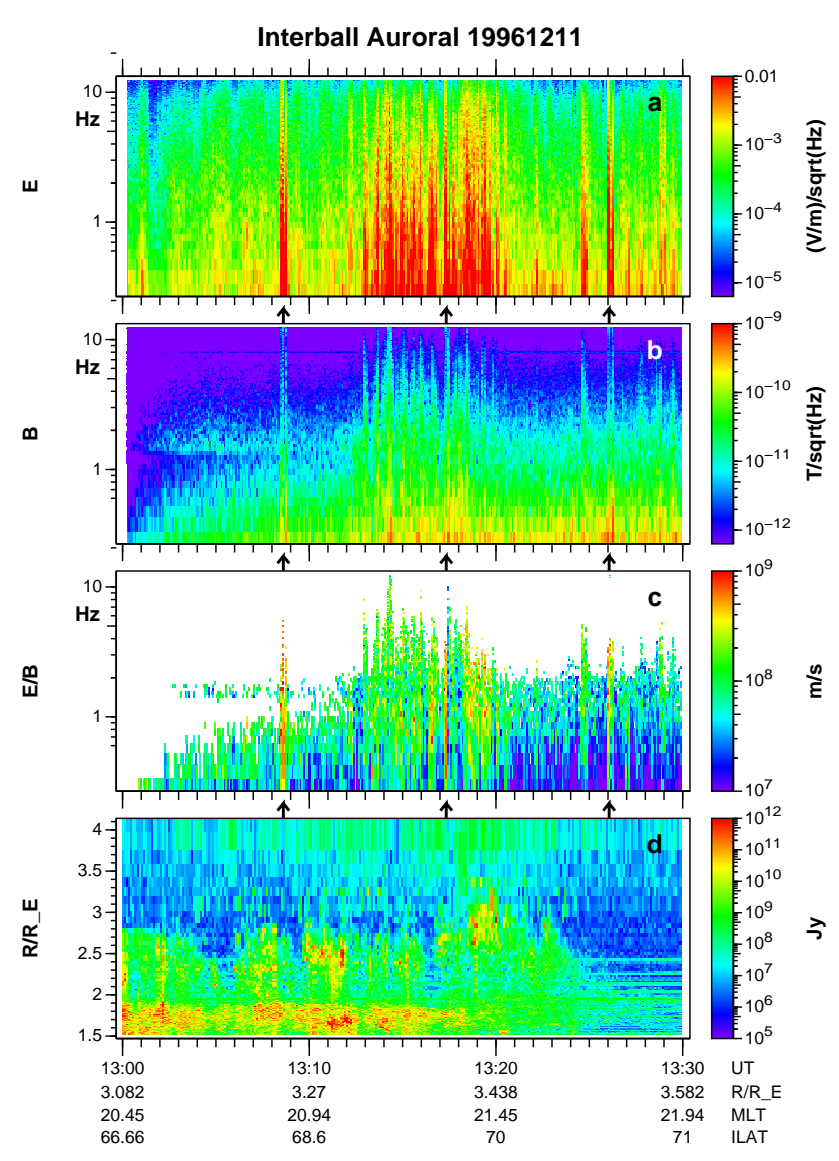

Fig. 8. Dot-AKR example event 11 December 1996 with IESP and POLRAD data, showing (a) electric IESP wave amplitude, (b) magnetic IESP wave power, (c) the $E / B$ ratio from IESP and (d) POLRAD AKR data. Artificial signals are shown by arrows. The intensity in panel (d) is in Jansky $\left(1 \mathrm{Jy}=10^{-26} \mathrm{~W} \mathrm{~m}^{-2} \mathrm{~Hz}^{-1}\right)$. The vertical axis in panel (d) is the radial distance corresponding to the AKR frequency assuming the frequency corresponding to the local electron gyrofrequency. The dot-AKR seen at 13:18-13:20 at $\sim 3 R_{E}$ radial distance $(45-70 \mathrm{kHz})$ occurs together with electromagnetic low-frequency wave activity. Dots at 13:02, 13:07-13:08 and 13:10-13:12 are not associated with low-frequency waves. Notice that perfect temporal correlation is not expected because AKR can propagate from remote sources; see Subsect. 6.2.

quency, $m_{e}$ the electron mass and $n$ the electron density (Stasiewicz et al., 2000). At 13:19, where the dot is seen in Fig. 8, the background magnetic field, $B_{0} \approx 13: 00 \mathrm{nT}$, so $v_{A}=3 \times 10^{7} \mathrm{~m} / \mathrm{s}$ if the plasma density is $n=1 \mathrm{~cm}^{-3}$ and the plasma consists of protons only. To get an idea of how sensitive the result is to different parameters, consider that since we are likely to be inside the auroral cavity, the density might be only $0.1 \mathrm{~cm}^{-3}$. This would increase $v_{A}$ to $10^{8} \mathrm{~m} / \mathrm{s}$, making most waves during the wave bursts in Fig. 8 Alfvénic. If the waves have a small perpendicular scale, even higher $E_{\perp} / B_{\perp}$ ratio waves may have an Alfvénic character. For example, for $n=0.1 \mathrm{~cm}^{-3}, \lambda_{e}=17 \mathrm{~km}$. If the perpendicular wavelength at the ionospheric footpoint is as small as $2 \mathrm{~km}$, it is $12 \mathrm{~km}$ at Interball altitude, in which case $\sqrt{1+k_{\perp}^{2} \lambda_{e}^{2}} \approx 9$, 
Table 1. Interball dot-AKR events

\begin{tabular}{|c|c|c|c|c|c|c|c|}
\hline Event & & $R / R_{E}$ & MLT & ILAT & Dot Freq & Gen. $R / R_{E}$ & $\mathrm{E} / \mathrm{B}$ \\
\hline 12 November 1996 & $13: 01-13: 02$ & 3.1 & 20.5 & 66.0 & $75-100$ & $2.4-2.7$ & $-1-$ \\
\hline 12 November 1996 & $13: 07-13: 08$ & 3.2 & 20.8 & 68.2 & $65-110$ & $2.3-2.8$ & $-1-$ \\
\hline 12 November 1996 & $13: 09-13: 11$ & 3.3 & 20.9 & 68.6 & $70-110$ & $2.3-2.7$ & $-1-$ \\
\hline 12 November 1996 & $13: 18-13: 20$ & 3.4 & 21.4 & 69.6 & $45-70$ & $2.7-3.2$ & $\mathrm{E} / \mathrm{B}$ \\
\hline 12 November 1996 & $18: 44-18: 55$ & 3.1 & 21.2 & 71.2 & $80-200$ & $1.9-2.6$ & $\mathrm{E} / \mathrm{B}$ \\
\hline 12 November 1996 & 19:00-19:01 & 3.3 & 22.0 & 72.5 & $60-85$ & $2.5-2.9$ & $-1-$ \\
\hline 12 November 1996 & $19: 35-19: 36$ & 3.8 & 23.9 & 73.2 & $65-160$ & $2.1-2.8$ & $-1-$ \\
\hline 12 November 1996 & $19: 37-19: 38$ & 3.8 & 0.0 & 73.1 & $55-70$ & $2.7-2.9$ & $-1-$ \\
\hline 12 November 1996 & $19: 55-19: 56$ & 3.9 & 0.7 & 72.3 & $65-90$ & $2.5-2.8$ & $-1-$ \\
\hline 16 December 1996 & $15: 10-15: 11$ & 3.7 & 22.2 & 71.3 & $40-55$ & $2.9-3.3$ & $-1-$ \\
\hline 16 December 1996 & $15: 13-15: 15$ & 3.7 & 22.3 & 71.4 & $50-70$ & $2.7-3.0$ & $\mathrm{E} / \mathrm{B}$ \\
\hline 16 December 1996 & $15: 55-15: 57$ & 4.0 & 0.0 & 70.8 & $50-70$ & $2.7-3.0$ & $-1-$ \\
\hline 16 December 1996 & $16: 00-16: 03$ & 4.0 & 0.2 & 70.6 & $50-70$ & $2.7-3.0$ & $-1-$ \\
\hline 16 December 1996 & $16: 04-16: 08$ & 4.0 & 0.4 & 70.2 & $50-70$ & $2.7-3.0$ & $\mathrm{E} / \mathrm{B}$ \\
\hline 16 December 1996 & $16: 11-16: 13$ & 4.0 & 0.6 & 69.8 & $30-50$ & $3.0-3.6$ & $\mathrm{E} / \mathrm{B}$ \\
\hline 18 December 1996 & $18: 57-19: 03$ & 3.4 & 21.7 & 73.5 & $55-100$ & $2.4-2.9$ & $-1-$ \\
\hline 18 December 1996 & $19: 18-19: 32$ & 3.7 & 23.1 & 73.8 & $45-130$ & $2.2-3.2$ & $\mathrm{E} / \mathrm{B}$ \\
\hline 19 December 1996 & $16: 30-16: 31$ & 4.0 & 22.1 & 69.6 & $40-50$ & $3.0-3.3$ & $\mathrm{E} / ?$ \\
\hline 2 February 1997 & $16: 19-16: 22$ & 4.0 & 22.0 & 69.5 & $40-100$ & $2.4-3.3$ & $\mathrm{E} / ?$ \\
\hline 2 February 1997 & $16: 23-16: 25$ & 4.0 & 21.3 & 69.1 & $60-100$ & $2.4-2.9$ & $\mathrm{E} / ?$ \\
\hline 2 February 1997 & $16: 26-16: 28$ & 4.0 & 21.4 & 68.8 & $60-100$ & $2.4-2.9$ & $-/ ?$ \\
\hline 9 October 1997 & $18: 23-18: 26$ & 3.5 & 20.8 & 75.5 & $80-140$ & $2.2-2.6$ & $\mathrm{E} / ?$ \\
\hline 9 October 1997 & $18: 34-18: 38$ & 3.6 & 21.5 & 75.1 & $45-120$ & $2.3-3.2$ & $\mathrm{E} / ?$ \\
\hline 9 October 1997 & $18: 43-18: 44$ & 3.7 & 21.9 & 74.7 & $95-145$ & $2.1-2.5$ & $\mathrm{E} / ?$ \\
\hline 9 October 1997 & $18: 49-18: 51$ & 3.8 & 22.2 & 74.3 & $45-75$ & $2.7-3.2$ & $\mathrm{E} / ?$ \\
\hline 9 October 1997 & $19: 02-19: 09$ & 3.9 & 22.7 & 73.2 & $40-90$ & $2.5-3.3$ & $-/ ?$ \\
\hline 9 December 1997 & $15: 03-15: 04$ & 3.5 & 22.0 & 70.3 & $75-90$ & $2.5-2.7$ & $-1-$ \\
\hline 1 November 1997 & $18: 22-18: 23$ & 3.7 & 22.6 & 63.7 & 60 & 2.9 & $-/ ?$ \\
\hline 1 November 1997 & $18: 22-18: 23$ & 3.7 & 22.6 & 63.7 & 30 & 3.6 & $-/ ?$ \\
\hline
\end{tabular}

Table 2. Interball SLFAKR events.

\begin{tabular}{|c|c|c|c|c|c|c|c|}
\hline Event & & $R / R_{E}$ & MLT & ILAT & Dot Freq & Gen. $R / R_{E}$ & $\mathrm{E} / \mathrm{B}$ \\
\hline 5 December 1996 & $12: 17-12: 18$ & 3.1 & 20.9 & 67.5 & $160-330$ & $1.6-2.1$ & $-1-$ \\
\hline 5 December 1996 & $12: 19-12: 22$ & 3.2 & 21.0 & 68.1 & $110-230$ & $1.8-2.3$ & $-1-$ \\
\hline 5 December 1996 & $12: 24-12: 26$ & 3.3 & 21.3 & 69.0 & $75-240$ & $1.8-2.7$ & $-1-$ \\
\hline 10 December 1996 & $19: 48-19: 56$ & 3.3 & 22.2 & 73.5 & $60-250$ & $1.8-2.9$ & $\mathrm{E} / \mathrm{B}$ \\
\hline 22 December 1996 & 10:02-10:08 & 3.6 & 20.6 & 73.0 & $55-110$ & $2.3-2.9$ & $\mathrm{E} / ?$ \\
\hline 28 January 1997 & $20: 31-20: 49$ & 4.0 & 22.1 & 72.3 & $50-160$ & $2.1-3.0$ & $-/ ?$ \\
\hline 9 February 1997 & $16: 01-16: 08$ & 4.0 & 20.5 & 70.2 & $85-110$ & $2.3-2.5$ & $-/ ?$ \\
\hline 9 February 1997 & $16: 23-16: 27$ & 4.0 & 21.0 & 68.4 & $90-180$ & $2.0-2.5$ & $\mathrm{E} / ?$ \\
\hline 15 February 1997 & $16: 04-16: 15$ & 3.9 & 18.9 & 79.6 & $70-170$ & $2.0-2.7$ & $-/ ?$ \\
\hline 15 February 1997 & $11: 00-11: 06$ & 4.0 & 0.1 & 72.5 & $50-130$ & $2.2-3.0$ & $\mathrm{E} / ?$ \\
\hline 9 September 1997 & $11: 08-11: 20$ & 4.0 & 0.5 & 71.5 & $50-110$ & $2.3-3.0$ & $\mathrm{E} / ?$ \\
\hline 15 September 1997 & $16: 50-17: 00$ & 4.0 & 1.1 & 68.0 & $60-300$ & $1.7-2.9$ & $\mathrm{E} / ?$ \\
\hline 21 September 1997 & $17: 10-17: 14$ & 4.0 & 0.0 & 70.0 & $60-130$ & $2.2-2.9$ & $\mathrm{E} / ?$ \\
\hline 8 October 1997 & $07: 19-08: 29$ & $3.1-3.9$ & $17.4-21.2$ & $69.6-74.3$ & $80-550$ & $1.4-2.6$ & $\mathrm{E} / ?$ \\
\hline 27 October 1997 & $22: 15-22: 50$ & $3.5-3.9$ & $22.8-23.3$ & $64.8-70.3$ & $65-700$ & $1.3-2.8$ & $\mathrm{E} / ?$ \\
\hline
\end{tabular}




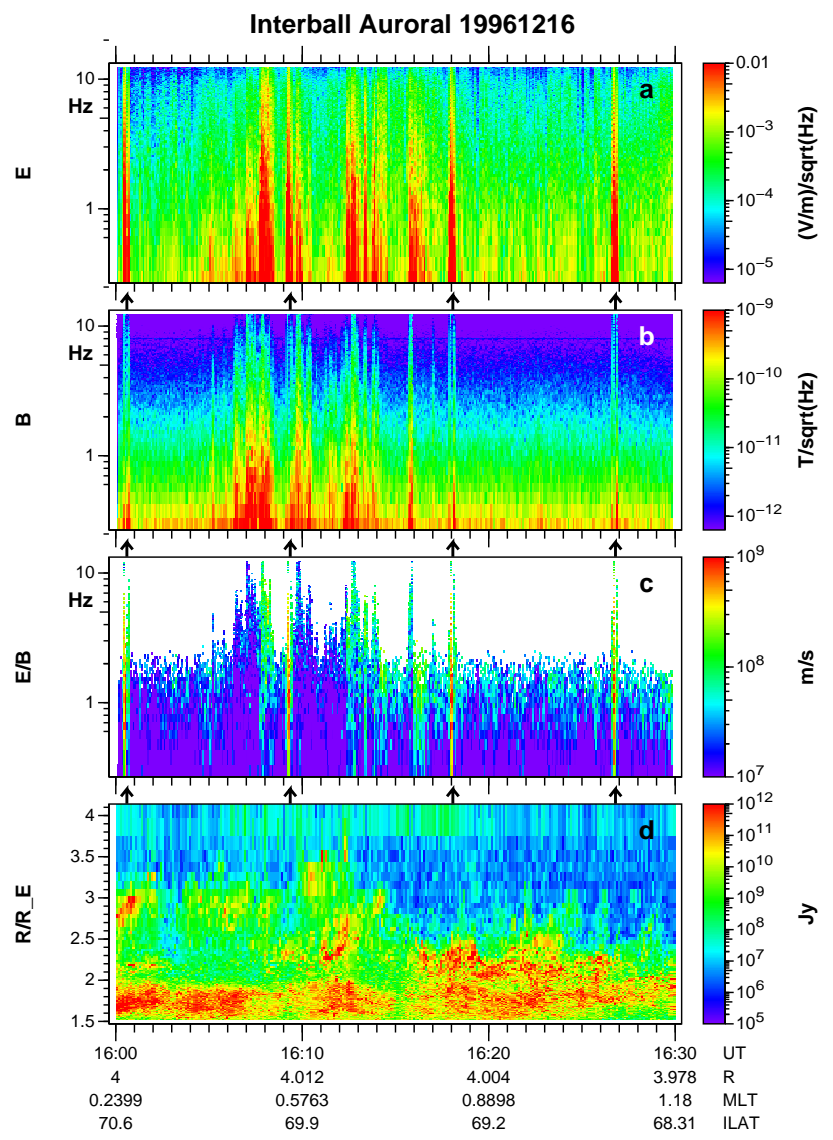

Fig. 9. Same as Fig. 8 but for 16 December 1996. Here the magnetic amplitude of the low-frequency waves is stronger than in the 11 December event (Fig. 8) and, consequently, the $E / B$ ratio (panel (c)) is smaller. Therefore, the correspondence between waves and dot-AKR is most easily seen by comparing panels (b) (magnetic amplitude) and (d) (dot-AKR). The dot seen at 16:12-16:13 at 3$3.6 R_{E}$ radial distance $(30-50 \mathrm{kHz}$ ) occurs (partly) simultaneously with low-frequency waves, while the dot at 16:00-16:03 does not. Again, because AKR can propagate from remote sources, perfect correlation is not expected.

giving the $E_{\perp} / B_{\perp}$ ratio a value of almost $10^{9}$. Thus, with this last set of parameters, almost all waves present in Fig. 8, panel (c) would be Alfvénic. The fact that the $E_{\perp} / B_{\perp}$ ratio in panel (c) of Fig. 8 increases with frequency is consistent with the interpretation that these are inertial Alfvén waves: usually one can assume that $k_{\perp}$ increases with $\omega$ and if so, the factor $\sqrt{1+k_{\perp}^{2} \lambda_{e}^{2}}$ and thus the $E_{\perp} / B_{\perp}$ ratio increases with frequency. The other event (Fig. 9) shows similar behaviour; there the satellite is at higher radial distance and the background magnetic field is $\sim 1.7$ times smaller than in Fig. 8 . Thus, we conclude that the data are consistent with the interpretation that the wave bursts in these examples are inertial Alfvén waves. In the events where we do not have the magnetic wave component measured, we obviously cannot make judgements about the Alfvénic character. However, the most natural assumption is that these low-frequency events behave similarly to those where magnetic field measurements are available. Under this assumption, all our data are consistent with the interpretation that the wave bursts are Alfvénic or contain a mixture of Alfvén and more electrostatic modes.

\section{Summary of observations}

We first briefly summarise our main observational results and discuss them further below:

1. There are more LFAKR (both dot-AKR and SLFAKR) emission events during wintertime and equinoxes than during summertime.

2. The dot-AKR emission radial distance peaks at $\sim 2.75 R_{E}$ during wintertime and at $\sim 3.25 R_{E}$ during summertime.

3. The low-frequency wave data are consistent with the interpretation that Alfvén wave activity is present during the dot-AKR and SLFAKR emissions.

\section{Discussion}

\subsection{Seasonal shift}

Many statistical measurements of auroral plasma physical parameters display a seasonal shift in altitude: all phenomena related to the electric field and plasma density structures are raised to higher altitude when the underlying ionosphere is sun-illuminated (Olsson and Janhunen, 2003). A similar trend is typically seen both when the data are binned according to the footpoint illumination condition and according to the season (summer/winter months). In the auroral density depletions the radial shift is about $0.5 R_{E}$ (Janhunen et al., 2002a). The existence of a seasonal shift is itself not a new result, but if a new parameter displays a similar shift, the indication is that the parameter is somehow physically related to the plasma density and/or static electric field structures. The underlying reason for the seasonal shifts is probably the increased ionospheric plasma density due to photoionisation when the ionosphere is sunlit: it is more difficult for magnetospheric processes to "dig" deep cavities in a more dense plasma during summertime, thus the cavities remain at higher altitude. Strong auroral electric fields follow a similar trend, since strong fields may only exist when there are not enough cold electrons to short-circuit them.

The seasonal shift seen in the altitude of dot-AKR emissions is more interesting. It indicates that the dot-AKR emissions probably are related to plasma density and/or static electric field structures $\sim 1.5 R_{E}$ above the bottom of the acceleration region. A corresponding shift is not seen in SLFAKR emission altitude, but the amount of data we have is not sufficient for discussing possible reasons for this.

It is interesting that the dot-AKR events are less common during summertime than during equinox and winter. The difference is not likely to be in the strength of the magnetospheric driver, but in the local plasma conditions. During 
summertime the plasma density is higher and deep cavities are less common (Janhunen et al., 2002a). This is another indication that the dot-AKR emissions are physically related to auroral cavities. Actually this is not very surprising, since the ordinary AKR in general is related to low plasma densities (Benson and Calvert, 1979; Hilgers, 1992).

\subsection{Association with low-frequency waves}

We pointed out that in about $50 \%$ of the dot-AKR and SLFAKR events significant low-frequency wave activity occurs simultaneously when the AKR is detected by Interball. It is natural that in some cases simultaneous low-frequency waves are not detected, since when detecting the AKR Interball need not be on the same field line where the AKR emission was generated (Fig. 10). Let $\Delta R$ denote the radial distance difference between Interball and the emission region. The AKR emission cone width is of the order of $45^{\circ}$, so the radiation may travel a perpendicular distance which is comparable to $\Delta R$. The median value of $\Delta R$ for the dot-AKR events of Table 1 is $0.85 R_{E}$, i.e. $5400 \mathrm{~km}$ perpendicular spacing at $R \approx 3.7 R_{E}$ which is the median radial distance of Interball for the events in Table 1. The scaling factor to the ionosphere is approximately the square root of the magnetic field ratio, i.e. about $3.7^{(3 / 2)} \approx 7$. Thus, Interball may start seeing dot-AKR emission when its footpoint is $5400 / 7 \approx 800 \mathrm{~km}$ away from the footpoint of the dot-AKR emission region. We have selected out events so that the footpoint of Interball is within the auroral bulge as seen by UVI and this may actually limit the perpendicular distance to the emission region more than the above rough calculation, since typically the bulge diameter is $\sim 1000 \mathrm{~km}$. However, it is likely that not the whole auroral bulge but only those parts of it that contain the most active and bright auroras contain the strongest Alfvén waves. Taking all of this together, we think that the fact that waves are seen in about $50 \%$ of the cases is not unexpected and is consistent with the assumption that such waves might exist in all cases if the satellite were on the same field line as the dot-AKR source region.

6.3 How Alfvén waves could accelerate electrons in a cavity

We now discuss the Alfvén wave scenario more closely. The dispersion relation of an inertial Alfvén wave is

$\omega=\frac{k_{\|} v_{A}}{\sqrt{1+k_{\perp}^{2} \lambda_{e}^{2}}}$

(Stasiewicz et al., 2000) if one ignores ion Larmor radius effects. The $E_{\|} / E_{\perp}$ ratio of the wave is

$\frac{E_{\|}}{E_{\perp}}=\frac{k_{\|} k_{\perp} \lambda_{e}^{2}}{1+k_{\perp}^{2} \lambda_{e}^{2}}$.

Solving Eq. (2) for $k_{\|}$and substituting in Eq. (3) yields

$\frac{E_{\|}}{E_{\perp}}=\frac{\omega k_{\perp} \lambda_{e}^{2}}{v_{A} \sqrt{1+k_{\perp}^{2} \lambda_{e}^{2}}}$.

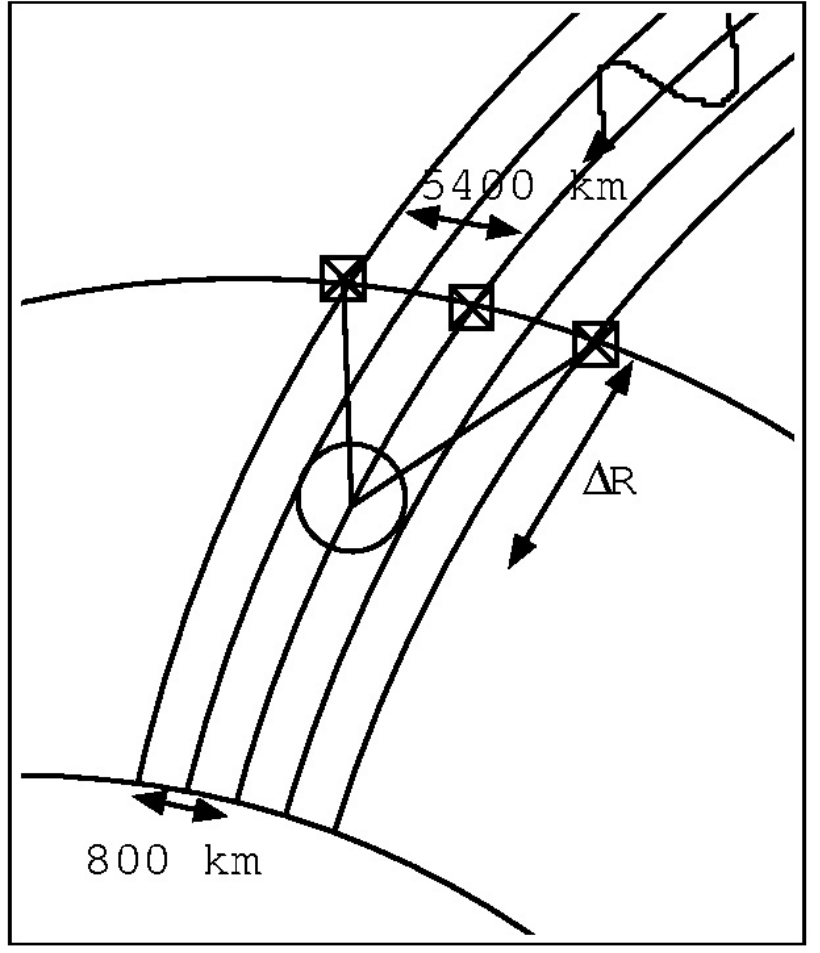

Fig. 10. The dot-AKR generation region (circle) may send AKR to any of the depicted Interball positions, although the Alfvén waves probably exist only on the flux tube of the generation region; thus, the satellite need not necessarily detect Alfvén waves when it detects dot-AKR even if Alfvén waves and dot-AKR always occur together.

If the perpendicular wavelength is not too small, $k_{\perp} \lambda_{e}<1$ and we have $E_{\|} / E_{\perp} \approx \omega k_{\perp} \lambda_{e}^{2} / v_{A}$. Since $v_{A} \sim 1 / \sqrt{n}$ and $\lambda_{e}^{2} \sim 1 / n$, the density dependence is $1 / \sqrt{n}$, i.e. $E_{\|} / E_{\perp}$ grows with decreasing density. Thus, if an Alfvén wave with suitable $k_{\perp}$ enters a density cavity, its parallel electric field component increases. This is one mechanism by which the dotAKR emission may occur: if there is a pre-existing density cavity into which a strong Alfvén wave enters from the magnetosphere, the parallel component may become large enough inside the cavity that electrons are accelerated enough to produce AKR emission. This is consistent with the fact that statistically, the auroral cavity occurrence frequency peaks at the same radial distance $2.75 R_{E}$ (Fig. 11, bottom panel; we do not consider the prominent $4.75 R_{E}$ feature now) where the dot-AKR emissions usually originate (Fig. 5, panel (b)). Notice that Fig. 11 shows only cases where the underlying Polar satellite footpoint is not illuminated by the Sun, which corresponds to the majority of the cases where dot-AKR is found, i.e. winter months (Fig. 5, panel (b)). Notice also that with Polar/PWI, one cannot determine which flux tubes are emitting the AKR, so the footpoint illumination condition based method could not be used in Fig. 5 but the month-based method is the only possibility. Figure 11, was produced by using the same Polar electric field data and analysis methods that are described by Janhunen et al. 
EFI/SCpot MLT=18..02, ILAT=65..74, Darkness
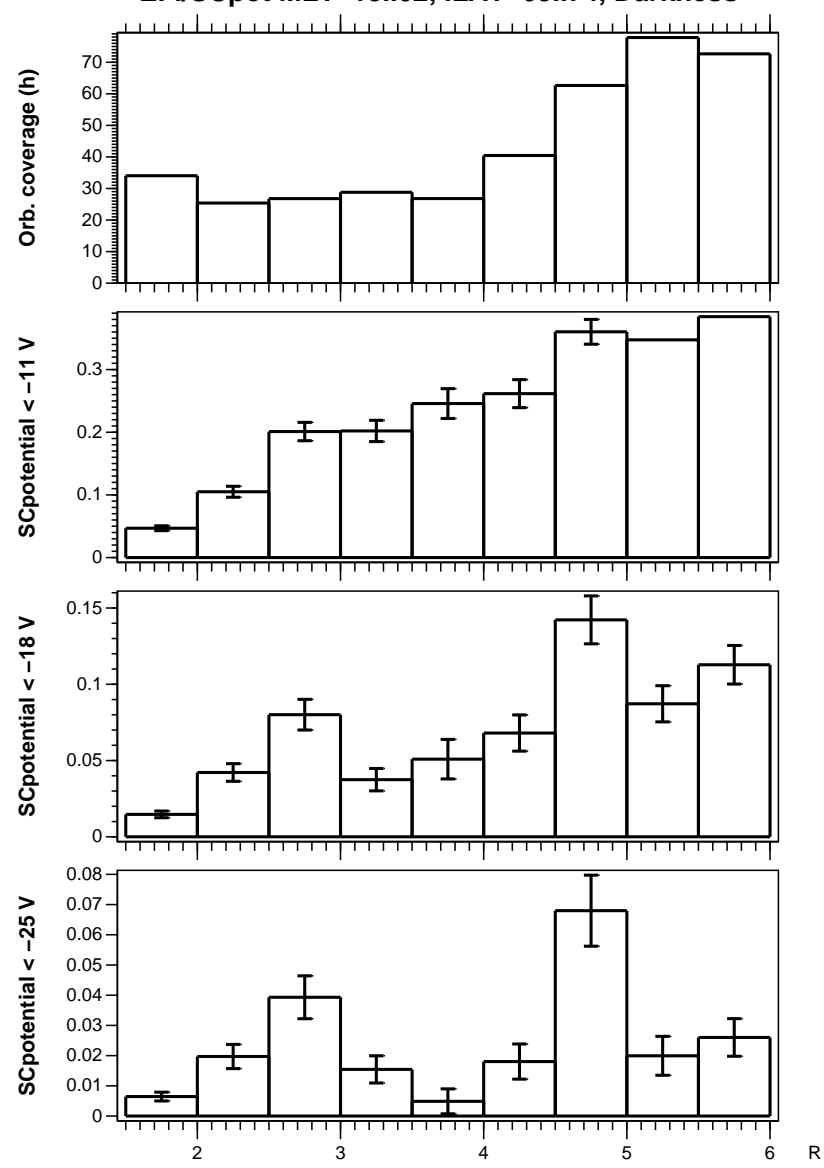

Fig. 11. The cavity occurrence frequency for $K_{p}>2$ (i.e. times when substorms are common) in the evening and midnight sectors (18:00-02:00 MLT) for three different spacecraft potential thresholds using Polar/EFI data (three lower panels), and the orbital coverage (top panel) when the ionospheric footpoint is not illuminated by Sun. The interesting double-peaked structure emerges as the spacecraft potential threshold is made more negative: it is most visible in the bottom panel. The occurrence frequency is the fraction of time the given condition is fulfilled; it is thus a number between 0 and 1. For more details, see Janhunen et al., (2002a).

(2002a), and shows only results for $K_{p}$ values larger than 2 to make the data set more representative for substorm periods. In Fig. 11 bottom panel, another cavity island (enhanced occurrence frequency of low plasma densities) is seen at high altitude at $4.75 R_{E}$ radial distance, i.e. the cavity occurrence frequency has a double-peaked structure. The physical explanation behind the $4.75 R_{E}$ feature is completely different from what we are considering here and therefore the discussion of the $4.75 R_{E}$ feature is given elsewhere; Janhunen et al. (2004b), see also Wygant et al. (2002).

The normal AKR originated from altitudes below the peak of the cavity occurrence, as seen by comparison of Figures 2 and 11. A reason for this may be found in the temporal evolution cycle of auroral cavities (Janhunen and Olsson, $2002 b$ ). When the process causing an auroral arc begins, a cavity starts to form (a "live" cavity). If the process main- taing the arc stops, electric fields and AKR emissions cease quickly in the electron time scale, but the cavity remains for $\sim 10$ min because it can be filled only in the ion time scale (a "dead" cavity). Because the plasma that refills the cavity comes mainly from the ionosphere, the lower part of the cavity disappears more rapidly. Thus, dead cavities are on the average found at higher altitude than live cavities. Since only live cavities emit normal AKR, it follows that the normal AKR emission altitude is lower than the most common cavity occurrence altitude (the latter contains both live and dead cavities). In our scenario, the Alfvén mechanism works equally well for live and dead cavities and thus no statistical distortion in the generation altitude of the dot-AKR emission is expected with respect to the peak cavity occurrence. This is in accordance with our data, since, as pointed out above, panel (b) of Fig. 5 and Fig. 11 show the same peak radial distance of $2.75 R_{E}$.

Another mechanism for accelerating electrons involving a density cavity and Alfvén wave has been proposed by Génot et al. (2000). In their model the important factor is the perpendicular gradient at the boundary of the cavity. In both mechanisms the result is an increase of the parallel electric field component because of the cavity. The Génot et al. (2000) mechanism is, however, related to the simpler one described above, since their model obeys a relationship approximately similar to Eq. (3) but with the density gradient length at the cavity boundary $(1 / n)|\nabla n|$ substituted for $k_{\perp}$.

In their studies of the detailed temporal behaviour of the WBB-AKR, de Feraudy et al. (2001) and Hanasz et al. (2001) found that in many cases the onset of the emission propagates so quickly in frequency space (or, equivalently, in altitude space) that only an Alfvénic process is capable of driving it. This is further evidence that Alfvén waves must play a role in explaining the dot-AKR.

\section{Conclusions}

With an underlying assumption that the cyclotron maser mechanism is the final step producing all types of AKR, we propose that Alfvén waves hitting a pre-existing deep density cavity are the mechanism specifically generating the dotAKR emission. In dot-AKR generation, electrons are transiently accelerated in the parallel direction since the parallel Alfvén wave electric field is locally enhanced due to the low plasma density found in a deep cavity. This interpretation is consistent with both statistical results (wintertime, i.e. low density preference for dot-AKR) and individual events (relation of dot-AKR to substorm onsets and simultaneous observation of Alfvén waves). Thus, the dot-AKR mechanism is different from normal AKR, which is thought to be generated by non-transient potential structure type electron acceleration (or Alfvénic acceleration caused by non-transient Alfvén-waves; the question as to what extent potential structure and Alfvénic acceleration can be distinguished at low altitude is somewhat unclear (Lysak, 1998)). The term "normal AKR" here does not include wide band bursts of AKR 
(Hanasz et al., 2001) which are probably transient Alfvénic in origin; their relationship to normal AKR and dot-AKR remains a topic for future research. If this interpretation is correct, the dot-AKR emission acts as a remote diagnostic indicator for transient Alfvén-wave induced electron acceleration in deep cavities. Although the dot-AKR phenomenon has thus far been reported only in the Earth's magnetosphere; in principle the mechanism could also work in other planetary magnetospheres.

Acknowledgements. The authors are grateful to G. Parks for making their Polar UVI data available. The work of AO was supported by the Swedish Research Council and that of PJ by the Academy of Finland. JH acknowledges the support of the Polish Committee of Scientific Research grant no: 5T12 00122.

Topical Editor in chief thanks two referees for their help in evaluating this paper

\section{References}

Bahnsen, A., Pedersen, B. M., Jespersen, M., Ungstrup, E., Eliasson, L., Murphree, J. S., Elpinstone, R. D., Blomberg, L., Holmgren, G. and Zanetti, L. J.: Viking observations at the source region of auroral kilometric radiation, J. Geophys. Res., 94, 66436654, 1989.

Benediktov, E. A., Getmantsev, G. G., Sazonov, Yu. A., and Tarasov, A. F.: Preliminary results of measurement of the intensity of distributed extraterrestrial radio-frequency emission at 72 and $1525 \mathrm{kC}$ frequencies by the satellite Electron 2, Cosmic Research, 36, 6, 791-794, 1965.

Bennett, E. L., Temerin, M., and Mozer, F. S.: The distribution of auroral electrostatic shocks below 8000-km altitude, J. Geophys. Res., 88,, 7107-7120, 1983.

Benson, R. F. and Calvert, W.: ISIS 1 observations at the source of auroral kilometric radiation, J. Geophys. Res., 94, 6643-6654, 1979.

Calvert, W.: The auroral plasma cavity, Geophys. Res. Lett., 8, 919921, 1981a.

Calvert, W.: The AKR emission cone at low frequencies, Geophys. Res. Lett., 8, 1159-1162, 1981b.

Chaston, C. C., Bonnell, J. W., Peticolas, L. M., Carlson, C. W. and McFadden, J. P.: Driven Alfvén waves and electron acceleration: a FAST study, Geophys. Res. Lett., 29, 11, 10.1029/2001GL013842, 30-1, 2002.

de Feraudy, H., Hanasz, J., Schreiber, R., Parks, G., Brittnacher, M., Perraut, S., Sauvaud, J. A., Lefeuvre, F., and Mogilevsky, M.: AKR bursts and substorm field line excitation, Phys. Chem. Earth, 26, 151-159, 2001.

Génot, V., Louarn, P., and Mottez, F.: Electron acceleration by Alfvén waves in density cavities, J. Geophys. Res., 105, 27 611$27620,2000$.

Filbert, P. C. and Kellogg, P. J.: Observations of low-frequency radio emissions in the Earth's magnetosphere, J. Geophys. Res., 94, 8867-8885, 1989.

Gurnett, D. A.: The Earth as a radio source: Terrestrial kilometric radiation, J. Geophys. Res., 79, 4227-4238, 1974.

Gurnett, D. A.: The Earth as a radio source: the nonthermal continuum, J. Geophys. Res., 80, 2751-2763, 1975.

Gurnett, D. A.: Auroral plasma waves, in Auroral physics, ed. by C.-I. Meng, M.J. Rycroft and L.A. Frank, Cambridge University Press, 1991.
Gurnett, D. A., Persoon, A. M., Randall, R. F., Odem, D. L., Remington, S. L., Averkamp, T. F., Debower, M. M., Hospodarsky, G. B., Huff, R. L., and Kirchner, D. L. et al.: The Polar Plasma Wave Instrument, Space Sci. Rev., edited by C. T. Russell, Kluwer Acad., 71, 597-622, 1995.

Hasegawa, A.: Particle acceleration by MHD surface wave and formation of aurora, J. Geophys. Res., 81, 5083-5090, 1976.

Hashimoto, K., Kudo, S., and Matsumoto, H.: Source of auroral myriametric radiation observed with Geotail, J. Geophys. Res., 103, 23 475-23 483, 1998.

Hui, C.-H. and Seyler, C. E.: Electron acceleration by Alfvén waves in the magnetosphere, J. Geophys. Res., 97, 3953-3963, 1992.

Hanasz, J., Krawczyk, Z., Mogilevsky, M. M., Schreiber, R., de Feraudy, H., Dudzinski, K., Romantsova, T. V., Nowakiewicz, W., Kraynyuk, A., Barylko, M., Buczkowska, A., Juchniewicz, J., Nazarov, V. N., Mikhalev, N.: Observations of auroral kilometric radiation on board Interball-2: Polrad experiment, Cosmic Res., 36, Engl. Transl., 575-586, 1998.

Hanasz, J., de Feraudy, H., Schreiber, R., Parks, G., Brittnacher, M., Mogilevsky, M. M., and Romantsova, T. V.: Wideband bursts of auroral kilometric radiation and their associated with UV auroral bulges, J. Geophys. Res., 106,, 3859-3871, 2001.

Hilgers, A.: The auroral radiating plasma cavities, Geophys. Res. Lett., 19, 237-240, 1992.

Imhof, W. L., Chenette, D. L., Datlowe, D. W., Mobilia, J., Walt, M. and Anderson, R. R.: The correlation of AKR waves with precipitating electrons as determined by plasma wave and X-ray image data from the POLAR spacecraft, Geophys. Res. Lett., 25, 289-292, 1998.

Janhunen, P., Olsson, A., and Laakso, H.: Altitude dependence of plasma density in the auroral zone, Ann. Geophys., 20, 17431750, 2002a.

Janhunen, P., Olsson, A., and Laakso, H.: A hybrid simulation model for a stable auroral arc, Ann. Geophys., 20, 1603-1616, 2002 b.

Janhunen, P., Olsson, A., and Laakso, H.: The occurrence frequency of auroral potential structures and electric fields as a function of altitude using Polar/EFI data, Ann. Geophys.,, 22, 951-970, 2004a.

Janhunen, P., Olsson, A., Hanasz, J., Russell, C. T., Laakso, H., and Samson, J. C.: Different Alfvén wave acceleration processes of electrons in substorms at $\sim 4--5 R_{E}$ and $2--3 R_{E}$ radial distance, Ann. Geophys., 22, 2213-2227, 2004b.

Keiling, A., Wygant, J. R., Cattell, C., Peria, W., Parks, G., Temerin, M., Mozer,F. S., Russell, C. T. and Kletzing, C. A.: Correlation of Alfvén wave Poynting flux in the plasma sheet at 4-7 $R_{E}$ within ionospheric electron energy flux, J. Geophys. Res., 107, SMP 24-1, CiteID 1132, DOI 10.1029/2001JA900140, 2002.

Keiling, A., Wygant, J. R., Cattell, C. A., Mozer, F. S., and Russell, C. T. : The global morphology of wave Poynting flux: powering the aurora, Science, 299, 383-386, 2003.

Kumamoto, A., Ono, T., and Iizima, M.: Seasonal and solar cycle variations of the vertical distribution of the occurrence probability of auroral kilometric radiation sources and of upflowing ion events, J. Geophys. Res., 108, A1, 1032, doi:10.1020/2002JA009522, 2003.

Kurth, W. S., Baumback, M. M., and Gurnett, D. A.: Directionfinding measurements of auroral kilometric radiation, J. Geophys. Res., 80, 2764-2770, 1975.

Louarn, P. and LeQueau, D.: Generation of the auroral kilometric radiation from plasma cavities: 1. Experimental study, Planet. Space Sci., 44, 199-210, 1996a. 
Louarn, P. and LeQueau, D.: Generation of the auroral kilometric radiation from plasma cavities: 2 . The cyclotron maser in small size sources, Planet. Space Sci., 44, 211-224, 1996 b.

Lysak, R. L.: The relationship between electrostatic shocks and kinetic Alfvén waves, Geophys. Res. Lett., 25, 2089-2092, 1998.

Lysak, R. L. and Lotko, W.: On the kinetic dispersion relation for shear Alfvén waves, J. Geophys. Res., 101, 5085-5094, 1996.

Olsson, A. and Janhunen, P.: Some recent developments in understanding auroral electron acceleration processes, IEEE Trans. Plasma Sci., 31, 6, 1178-1191, 2003.

Perraut, S., Roux, A., Darrouzet, F., de Villedary, C., Mogilevsky, M., and Lefeuvre, F.: ULF wave measurements onboard the Interball auroral probe, Ann. Geophys., 16,, 1105-1116, 1998.

Roux, A., Hilgers, A., de Feraudy, H., Le Queau, D., Louarn, P., Perraut, S., Bahnsen, A., Jespersen, M., Ungstrup, E., and Andre, M.: Auroral kilometric radiation sources - In situ and remote observations from Viking, J. Geophys. Res., 98,, 11 657-11 670, 1993.

Stasiewicz, K., Khotyaintsev, Y., Berthomier, M., and Wahlund, J.E.: Identification of widespread turbulence of dispersive Alfven waves, Geophys. Res. Lett., 27, 173-176, 2000.

Steinberg, J.-L., Hoang, S., and Bosqued, J.-M.: Isotropic terrestrial kilometric radiation: a new component of the Earth's radio emission, Ann. Geophys., 8,, 671-686, 1990.
Strangeway, R. J., Kepko, L., Elphic, R. C., Carlson, C. W., Ergun, R. E., McFadden, J. P., Peria, W. J., Delory, G. T., Chaston, C. C., Temerin, M. et al.: FAST observations of VLF waves in the auroral zone: evidence of very low plasma densities, Geophys. Res. Lett., 25,, 2013-2016, 1998.

Strangeway, R. J., Ergun, R. E., Carlson, C. W., McFadden, J. P., Delory, G. T., and Pritchett, E. L.: Accelerated electrons as the source of auroral kilometric radiation, Phys. Chem. Earth, 26, 1-3, 145-149, 2001.

Torr, M. R., Torr, D. G., Zukic, M., Johnson, R. B., Ajello, J., Banks, P., Clark, K., Cole, K., Keffer, C., and Parks, G. : A far ultraviolet imager for the International-Solar Terrestrial Physics Mission, Space Sci. Rev., 71, 329-383, 1995.

Volwerk, M., Louarn, P., Chust, T., Roux, A., de Feraudy, H., and Holback, B.: Solitary kinetic Alfvén waves: a study of the Poynting flux, J. Geophys. Res., 101, 13 335-13344, 1996.

Wu., C. S. and Lee, L. C.: A theory of the terrestrial kilometric radiation, . Astrophys. J., 230, 621-626, 1979.

Wygant, J. R., Keiling, A., Cattell, C. A., Lysak, R. L., Temerin, M., Mozer, F. S., Kletzing, C. A., Scudder, J. D., Streltsov, V., Lotko, W., Russell, C. T.: Evidence for kinetic Alfven waves and parallel electron energization at 4-6 $R_{E}$ altitudes in the plasma sheet boundary layer, J. Geophys. Res., 107, A8 10.1029/2001JA900113, 2002. 